\title{
A DISCIPLINA DE CÁLCULO NUMÉRICO E SUA RELAÇÃO COM A ENGENHARIA: APLICAÇÕES E POTENCIALIDADES
}

\author{
DOI: $10.37702 / 2175-957 X . C O B E N G E .2021 .3574$
}

Andromeda Goretti de Menezes Campos - andromeda.campos@ifes.edu.br Instituto Federal do Espírito Santo Rua Engenheiro Fábio Ruschi 405 29050-670 - Vitória - ES

Danieli Soares de Oliveira - danieli@ifes.edu.br Instituto Federal do Espírito Santo Campus Cariacica

Resumo: $O$ presente trabalho visa apresentar e discutir a importância da disciplina de cálculo numérico para alunos de graduação em engenharia, sobretudo em temas correlatos a formação dos discentes, visando mostrar as potencialidades deste conjunto de ferramentas/métodos usados para se obter a solução de problemas matemáticos de forma aproximada. Esses métodos se aplicam a problemas que não apresentam uma solução exata e que, por este motivo, precisam ser resolvidos numericamente. Em especial, este artigo apresenta experiências exitosas verificadas no curso de graduação em Engenharia de Produção do Instituto Federal do Espírito Santo - campus Cariacica. Grande parte dos problemas trabalhados nas disciplinas básicas e específicas dos cursos de graduação na área de engenharia surgem da observação da sociedade, da indústria, da natureza, dentre outros aspectos, e tais problemas podem ser descritos por meio de modelos matemáticos, modelos estes que devem ser muitas vezes modelados numericamente para a obtenção de resultados e conclusões. Neste trabalho serão apresentadas temáticas relacionadas a cálculo numérico $e$ outras disciplinas básicas do curso de engenharia. Alguns exemplos: soluções de mecânica dos fluidos e fenômenos de transporte; análise de dados ambientais, relacionados à disciplina de ciências do ambiente; e avaliação econômica $e$ financeira de projetos de engenharia. Em todos os trabalhos desenvolvidos foram realizadas atividades alternativas com os discentes utilizando tecnologias digitais e metodologias ativas, visando garantir que os estudantes se mantivessem motivados e estimulados para desenvolver as atividades propostas. Desta forma, espera-se com este trabalho incentivar outros docentes a propor atividades 
diversificadas relacionando os conteúdos de cálculo numérico com outras áreas de interesse dos alunos, diretamente relacionadas a sua área de formação, de forma a ampliar suas possibilidades de resolução de problemas e análise crítica.

Palavras-chave: Cálculo numérico. Ensino de engenharia. Metodologias ativas. Aprendizagem Baseada em Problemas. 


\section{A DISCIPLINA DE CÁLCULO NUMÉRICO E SUA RELAÇÃO COM A ENGENHARIA: APLICAÇÕES E POTENCIALIDADES}

\section{INTRODUÇÃO}

As mudanças na sociedade, indústria e serviços demandam novos requisitos e práticas mais eficazes, o que aumenta a exigência e o desafio dos profissionais. Cabe ao profissional lançar mão de sua capacidade de integrar seus conhecimentos, habilidades e competências para gerar soluções de problemas de naturezas distintas e de diferentes níveis de complexidades, em um curto espaço de tempo. Além disso, requer que sejam capazes de se adaptarem a situações inconstantes, diferentes e desconhecidas. Este contexto influencia, de forma expressiva, a prática do profissional de Engenharia (Lima et al., 2011; Lima et al., 2012).

Porém, há uma distância entre a formação em engenharia e as competências exigidas pelo mercado de trabalho (em especial a indústria de bens e serviços) e pela sociedade. Tradicionalmente, o formato do processo de ensino-aprendizagem é marcado pela posição central do professor, considerando-o detentor de todo o conhecimento, e colocando os estudantes como atores passivos do processo. É necessário que se faça uma transição entre o ensino-aprendizagem baseado na ideia de transmissão do conhecimento, para aquele baseado no desenvolvimento de competências, que possibilite ao futuro profissional obter a capacidade de mobilizar recursos de aprendizagem em contextos específicos, acadêmicos e/ou profissionais. Assim, tornando-o um processo ativo, o qual chama-se Aprendizagem Ativa.

Para cumprir com este objetivo, a Educação em Engenharia vem tomando novo formato, acompanhando estas mudanças, lançando mão de novas técnicas, métodos e objetos de aprendizagem com objetivo de estimular os alunos e assim desenvolver as competências necessárias para sua formação. Aquere et al. (2012), afirma que os novos formatos de ensino e aprendizagem sugerem que a coordenação de equipes de estudantes baseados em problemas ou projetos demandam novos papéis para os professores e alunos, incluindo a necessidade de maior autonomia, integração de conteúdo interdisciplinar e trabalho em equipe.

Desta forma, este artigo tem por objetivo apresentar experiências exitosas verificadas no curso de graduação em Engenharia de Produção do Instituto Federal do Espírito Santo - campus Cariacica, utilizando tecnologias digitais e metodologias ativas.

Para atingir este objetivo, alguns objetivos específicos são necessários:

- Realizar a revisão bibliográfica sobre o conteúdo relacionado à pesquisa;

- Coletar dados dos problemas resolvidos pelos alunos;

- Analisar os dados coletados;

- Apresentar as experiências realizadas.

\section{REVISÃO BIBLIOGRÁFICA}

Segundo Lima et. al (2017), o profissional de Engenharia é reconhecido pelas competências técnicas adquiridas e por serem aptos a projetar, operar, executar e gerenciar sistemas tecnológicos. Neste contexto, eles são capazes de aplicar conceitos matemáticos e 
científicos e ferramentas para identificar, formular e resolver problemas que contribuem positivamente para a sociedade. Além disso, os engenheiros devem ter um forte senso de interação humana, tanto ao projetar soluções para a sociedade quanto ao realizar planejamento, execução e gestão do desenvolvimento de soluções.

\subsection{COMPETÊNCIAS PARA UM PROFISSIONAL DE ENGENHARIA}

A acreditação americana ABET (Accreditation Board for Engineering and Technology) e a europeia EUR-ACE (European Accreditation Board for Engineering Education) para o credenciamento do Ensino em Engenharia, trazem novas skills para 0 profissional desta área. Estas skills estão atreladas aos desafios que o mundo atual impõe para este profissional. Competências que devem ser desenvolvidas durante o processo ensino e aprendizagem. São elas: capacidade de funcionar em equipes multidisciplinares; capacidade de identificar e resolver problemas de ciências aplicadas; compreensão da responsabilidade profissional e ética; capacidade de comunicação eficaz; educação ampla necessária para compreender o impacto das soluções em um contexto global e social; reconhecimento da necessidade e a capacidade de se envolver na aprendizagem ao longo da vida; conhecimento de questões contemporâneas; e capacidade de usar técnicas, habilidades e ferramentas científicas e técnicas modernas necessárias para a prática profissional.

No Brasil, a ABENGE (Associação Brasileira de Educação em Engenharia), traz as seguintes competências para o atendimento ao perfil do Engenheiro: identificar, formular e projetar soluções para problemas complexos (solução de problemas); projetar e conduzir a experimentação, analisar e interpretar dados e obter resultados (pesquisa); conceber, projetar e analisar sistemas, produtos, componentes ou processos (projeto); simular e analisar diferentes cenários com foco na tomada de decisões (gestão); comunicar-se efetivamente (comunicação); trabalhar e liderar equipes multidisciplinares (trabalho em equipe); interpretar e aplicar com ética a legislação e os atos normativos no âmbito do exercício da profissão (legislação e ética); reconhecer a necessidade de ensino / aprendizagem ao longo da vida profissional (atualização permanente); e competências específicas, em acordo com o curso de engenharia em termos de modalidade escolhida e características regionais demandadas (modalidade) (ABENGE, 2018).

É possível observar que as competências definidas pela ABENGE, estão de acordo com as competências definidas pela ABET e EUR-ACE.

\subsection{EDUCAÇÃO EM ENGENHARIA}

A educação em engenharia é a base para o desenvolvimento da sociedade. Sem inovações tecnológicas, não haverá produção de novos bens, nem crescimento econômico e nem desenvolvimento humano. As inovações fazem parte dos mecanismos de mercado para estabelecer novos mercados. Os mercados, exigem um rápido desenvolvimento de inovações e, portanto, de conhecimento tecnológico e científico (Kolmos, A., Dahms, M. e Du, X., 2012).

Para Kolmos, Dahms e Du (2012), isso cria muitos desafios para o ensino de engenharia, portanto, os autores trazem algumas afirmações que levam o Ensino em Engenharia a um processo de mudanças: 1. O conhecimento pode ficar desatualizado em 
alguns anos, o que cria desafios para o ensino de engenharia em todo o mundo; 2 . A inovação não se baseia mais no conhecimento individual, mas no conhecimento colaborativo; 3. A construção colaborativa do conhecimento está cada vez mais complexa. Por um lado, a globalização exige novas formas de compartilhar o trabalho, portanto, envolve a compreensão intercultural da colaboração internacional. Por outro lado, porque a complexidade envolve construções de conhecimento interdisciplinares; e 4. Há um grande desafio em tornar esse rápido desenvolvimento tecnológico muito mais sustentável em questões como meio ambiente e responsabilidade social.

\subsection{PROBLEM-BASED LEARNING (PBL)}

Os métodos de aprendizagem ativa consistem no fato do aluno ser o ator principal, ou seja, o responsável por sua própria aprendizagem, uma vez que o professor auxiliará na condução da construção do conhecimento, tendo um papel de "tutor", e avaliador do aprendizado do aluno.

A Aprendizagem Baseada em Problemas (PBL) consiste em um método de aprendizagem ativa que lança mão de problemas reais, servindo de estímulo para o desenvolvimento do pensamento crítico, de habilidades de resolução de problemas e da aprendizagem dos conceitos que integram o conteúdo programático (White, 1996). Para Maurer (2015, p. 372), "PBL se baseia na aprendizagem colaborativa, assumindo que a deliberação e a discussão aumentam o conhecimento e a compreensão". Já Boud e Feletti (1999) compreendem o PBL como um método que promove o desenvolvimento de habilidades, no qual ao início da aprendizagem se dá pela exposição de um problema, uma questão ou um enigma, estimulando a aprendizagem. Desta forma, a aprendizagem baseada em problemas propicia aos estudantes explorarem situações reais com ênfase na solução de problemas e no trabalho em equipe, desenvolvendo habilidades e competências educacionais que possibilitarão enfrentar o mundo em constante mudança. Para Christie e de Graaff (2017), o PBL se adaptou com o tempo e as mudanças para renovar no ensino de nível superior e fornecer uma avaliação de estudo de caso de aprendizagem.

Para Barrows (2010), uma intervenção educacional apenas será considerada PBL se contiver: apresentação do problema antes da teoria; problemas baseados no mundo real; elaboração de um produto concreto como, por exemplo, um relatório, um modelo, uma maquete, um desenho esquemático; método educacional centrado no estudante; aprendizagem em equipe; papel do professor como facilitador do processo; produto concreto do ciclo PBL (Figura 1). 
Figura 1 - Ciclo da Aprendizagem Baseada em Problemas

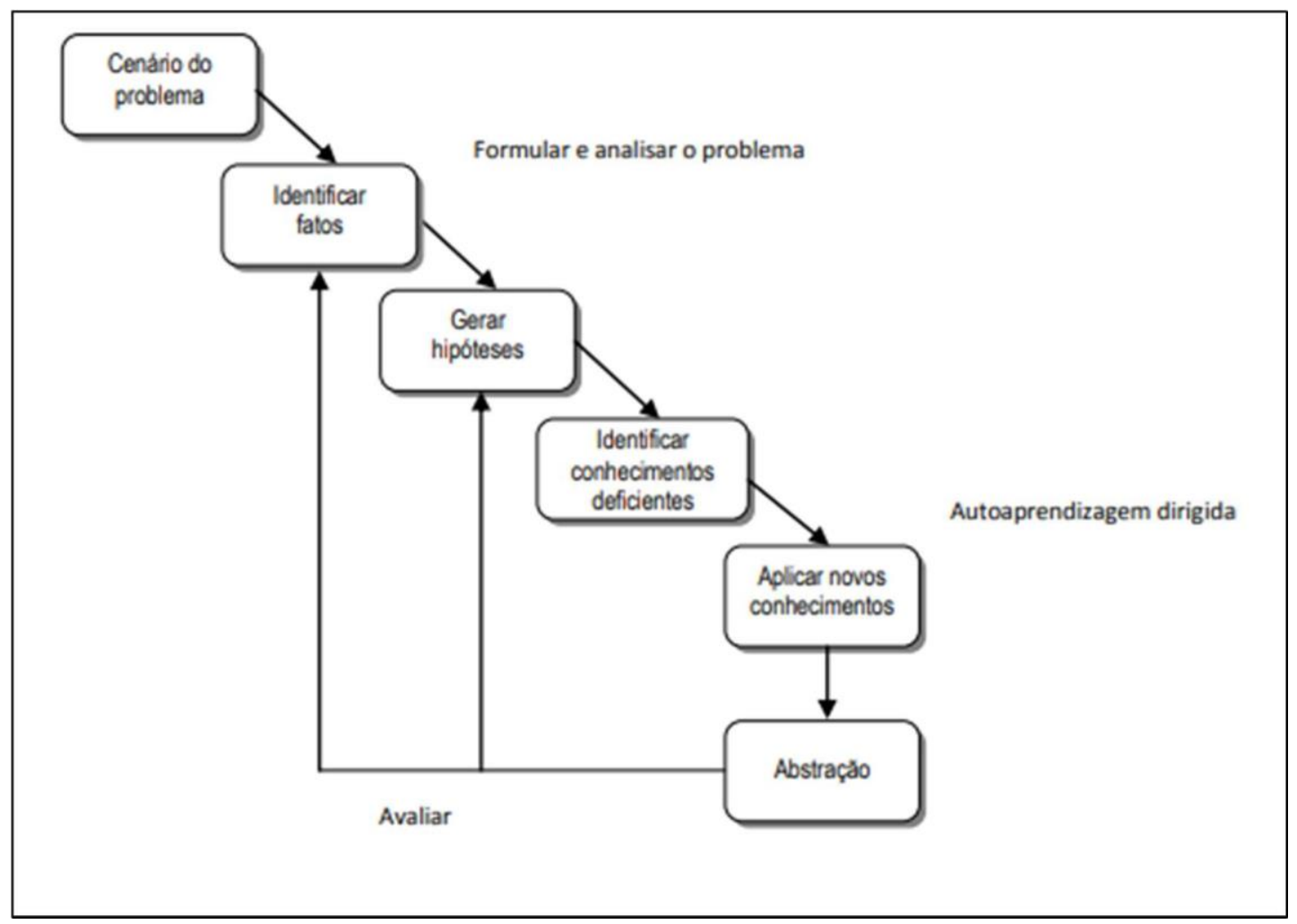

Fonte: HMELO-SILVER, 2004

No PBL, problemas complexos do mundo real são usados para motivar os estudantes na identificação e investigação dos princípios e conceitos necessários para a resolução dos problemas em toda a sua extensão. De acordo com Duch, Groh e Allen (2001), muitos dos resultados recomendados e desejáveis no ensino superior são alcançados pelo PBL, especialmente as habilidades para:

- pensar criticamente e ser capaz de analisar e resolver complexos problemas do mundo real;

- identificar, avaliar e utilizar recursos de aprendizagem apropriados;

- trabalhar cooperativamente em grupos;

- demonstrar habilidade de comunicação versátil e efetiva, tanto verbal quanto escrita;

- ter capacidade de síntese;

- utilizar o conteúdo de conhecimentos e as habilidades intelectuais adquiridas na universidade para tornarem-se contínuos aprendizes.

\section{METODOLOGIA}

O desenvolvimento deste trabalho foi dividido em duas etapas: revisão bibliográfica e relato de experiência.

Para o desenvolvimento da etapa de pesquisas bibliográficas foram realizadas buscas sobre temáticas relacionadas à pesquisa científica na graduação e iniciação científica na engenharia. Destaca-se que a instituição de ensino onde a pesquisa foi desenvolvida disponibiliza acesso aos conteúdos científicos por meio da Rede Mundial de Computadores. Inclusive, discentes e docentes podem usufruir desses acessos a partir da Rede Corporativa do Ifes ou remotamente por meio da Comunidade Acadêmica Federada 
(CAFe). As principais editoras e fornecedores de conteúdo científicos disponibilizados à comunidade acadêmica do Ifes são: Scopus, Springer, Wiley-Blackwell, Taylor \& Francis, Sage, IEEE, Oxford University Press, Cambridge University Press, Emerald, ScienceDirect, Elsevier, Ebsco Host, dot.lib, Alexander Street Press, Enciclopedia Britannica, HighWire Press, JournalCitationReports, ProQuest, Thomsom Reuters, Isi Web of Science.

Para a seção de relato de experiência foram utilizados os conhecimentos das autoras sobre os projetos desenvolvidos, tanto na disciplina de cálculo numérico quanto em outras disciplinas pertencentes à grade do curso de graduação. Os trabalhos abordados nesta pesquisa foram realizados em 2020/2021 com 48 discentes da disciplina de Cálculo Numérico, ofertada pelo curso de graduação em Engenharia de Produção do Instituto Federal do Espírito Santo, campus Cariacica.

\section{DESENVOLVIMENTO}

A primeira fase do desenvolvimento deste trabalho foi a revisão bibliográfica, que contou com os assuntos pertinentes ao seu desenvolvimento. Foram eles: Competências para um profissional de Engenharia; Educação em Engenharia; e Problem Based Learning (PBL). Esta revisão foi necessária para o desenvolvimento do planejamento da disciplina, de modo a contemplar o maior número possível de competências estabelecidas pela ABEPRO, quanto pelas $A B E T$ e EUR-ACE, visto que estamos inseridos em um contexto de globalização.

A segunda fase constitui-se no relato da experiência relativo a disciplina de Cálculo Numérico, do 5ำ período do curso de graduação em Engenharia de Produção, do Instituto Federal do Espírito Santo. Para essa disciplina foi utilizada uma mescla entre a metodologia tradicional e uma abordagem de Aprendizagem Ativa, especificamente Aprendizagem Baseada em Problemas (PBL). Os alunos trabalham em equipes com o intuito de solucionar problemas reais, encontrados em artigos científicos, dentro do tema geral "Aplicações na Engenharia".

Para desenvolver as soluções, as equipes de alunos articulam teoria e prática, com base nos Métodos Numéricos estudados e em um conjunto de conhecimentos interdisciplinares, como os que serão tratados neste trabalho: mecânica dos fluidos e fenômenos de transporte; análise de dados ambientais, relacionados à disciplina de ciências do ambiente; avaliação econômica e financeira de projetos de engenharia. Cada equipe modela o problema real e se utiliza do conhecimento adquirido em Cálculo Numérico e demais disciplinas, além de ferramentas computacionais para gerar a solução. Nesta disciplina utiliza-se a Linguagem $\mathrm{C}$ ou a que o aluno tiver o maior domínio. A escolha pela Linguagem $C$ é devido às demais disciplinas do curso de Engenharia de Produção, no contexto da computação, utilizarem esta linguagem; portanto, os alunos já têm experiência na mesma.

Apesar da disciplina já utilizar este método de trabalho desde 2018, nestes relatos de experiência serão descritas as experiências ocorridas no semestre de 2020/2, em um contexto de pandemia, no qual a disciplina foi realizada totalmente a distância. Apesar do curso ser Engenharia de Produção, as duas turmas foram compostas por alunos de outras Engenharias de outros Campi do Ifes. Os alunos dividiram-se em 12 equipes, com 4 alunos cada, em um total de 23 alunos de uma turma e 25 alunos da outra turma; porém os grupos poderiam ser formados com alunos de turmas distintas. Ao longo do semestre, além de atividades pontuais sobre cada Método Numérico em avaliações individuais, cada equipe desenvolveu dois trabalhos que foram apresentados em dois seminários ao longo do semestre letivo.

Inicialmente foi realizada a introdução sobre ideia central da disciplina, especialmente da sua importância dentro dos cursos de Engenharia, e uma explanação 
sobre diferentes problemas que poderiam ser resolvidos com o conteúdo da disciplina. Ou seja, houve uma contextualização, para que fosse possível gerar uma motivação inicial nos alunos. Em um segundo momento, houve uma explicação acerca das competências requeridas para um profissional de Engenharia, bem como aquelas que este método de ensino poderá contribuir para que adquiram. Em seguida, cada equipe recebeu as seguintes orientações sobre os trabalhos a serem desenvolvidos, e para realizá-los, os alunos utilizaram o Ciclo da Aprendizagem Baseada em Problemas, apresentado anteriormente.

Seminários de Cálculo Numérico: "Resolução de Problemas Reais de Engenharia".
Objetivo: Resolver problemas reais de Engenharia em equipe, de maneira a construir os
conhecimentos sobre os diversos métodos numéricos a serem estudados na disciplina de
Cálculo Numérico, bem como lançar mão de demais conhecimentos de outras disciplinas
estudadas no curso, para encontrar a melhor solução. Para isso, é necessário:
Objetivos Específicos:
- Encontrar um artigo científico que traga um problema real de Engenharia passível de ser
resolvido no contexto da disciplina de Cálculo Numérico;
- Realizar pesquisa bibliográfica sobre os temas relacionados ao problema;
- Analisar o problema sobre o ponto de vista do uso na profissão de Engenharia;
- Resolver o problema através da modelagem e implementação em uma solução na
Linguagem C;
- Utilizar um "Diário de Bordo" para anotar as intercorrências durante o processo, e as
soluções dadas a cada uma (individualmente e em equipe) - (Moodle);
- Comparar a velocidade de convergência entre os métodos como parte da descoberta da
solução ideal; e
- Apresentar os resultados em um relatório e seminário.
Equipe:
- Deve ser composta de no máximo 4 alunos (composição de livre escolha dos alunos da
turma, deve-se lembrar dos fatores afinidade, parceria, compromisso e outros).
Pontuação: 25.0 pontos (cada)
Datas de Entrega e Apresentação:
Seminário I: $26 / 01 / 2021$
Seminário II: $11 / 03 / 2021$
Conteúdo do Relatório Final:
- Referencial Teórico;
- Descrição e modelagem do problema;
- Justificativa do(s) método(s) escolhido(s) para a solução do problema;
- Demonstrar a solução, bem como o algoritmo utilizado para resolvê-lo;
- Descrever as competências técnicas, adquiridas em outras disciplinas, utilizadas para
encontrar a melhor solução;
- Descrever as competências para um profissional de Engenharia adquiridas/utilizadas; e
- Descrever as dificuldades encontradas durante o processo.

Para cada um dos objetivos específicos do trabalho, foi realizada a moderação por parte da professora, e em muitos casos, foi necessário que os alunos buscassem o apoio de outros professores da instituição, porém isso ficou sob a responsabilidade deles. Desta forma, a professora agiu como facilitadora do processo, não mostrando diretamente as soluções para seus questionamentos, mas sim orientando. 
Ao mesmo tempo que os alunos pesquisavam e buscavam as soluções para os problemas, o conteúdo da disciplina foi inserido e atividades avaliativas individuais foram realizadas. Conforme proposto por Quadros, R. e Villas-Boas. V. (2010), “ (...) a avaliação ocorreu em um processo contínuo, considerando a evolução e a construção da aprendizagem por parte dos estudantes. Foi analisada a participação, o engajamento e a contribuição de cada estudante para a resolução do problema proposto".

A seguir, estão listados os conteúdos programáticos da disciplina contemplado em cada um dos seminários:

1. Conteúdo do Seminário I:

- Método da Bissecção

- Método da Posição Falsa

- Método do Ponto Fixo

- Método de Newton-Raphson

- Método da Secante

2. Conteúdo do Seminário II:

- Método das Diferenças Divididas

- Método dos Quadrados Mínimos: Caso Discreto

- Método dos Quadrados Mínimos: Caso Contínuo

- Integração Numérica: Regra dos Trapézios

- Integração Numérica: Regra dos Trapézios Repetida

- Integração Numérica: Regra 1/3 de Simpson

- Integração Numérica: Regra 1/3 de Simpson Repetida

- Problemas de Valor Inicial: Métodos de Passo Simples (ou Passo Um)

- Problemas de Valor Inicial: Métodos de Passo Múltiplo

- Problemas de Valor Inicial: Métodos de Previsão-Correção

Os dados relativos a esta experiência, foram coletados através da observação de todo o processo de desenvolvimento da disciplina e dos relatórios finais de cada um dos trabalhos realizados pelos alunos.

No

Quadro 1 a seguir, estão descritos alguns problemas resolvidos pelos alunos, 0 método utilizado para a resolução do problema, bem como as respectivas disciplinas envolvidas:

Quadro 1- Problemas Resolvidos

\begin{tabular}{|c|c|c|}
\hline Problema 1 & Método Numérico & Disciplina \\
\hline $\begin{array}{l}\text { Estimar a produção de soja num } \\
\text { determinado período de tempo, } \\
\text { considerando valores anteriores já } \\
\text { determinados, para um melhor } \\
\text { planejamento. }\end{array}$ & $\begin{array}{l}\text { Quadrados } \\
\text { mínimos }\end{array}$ & $\begin{array}{lr}\text { Planejamento } & \mathrm{e} \\
\text { Controle } & \mathrm{da} \\
\text { Produção. } & \end{array}$ \\
\hline $\begin{array}{l}\text { Definição de preço adequado à venda: } \\
\text { buscar um preço para o produto que } \\
\text { maximize os lucros da empresa. }\end{array}$ & $\begin{array}{l}\text { Newton e Ponto } \\
\text { Fixo }\end{array}$ & $\begin{array}{l}\text { Economia } \\
\text { Engenharia }\end{array}$ \\
\hline $\begin{array}{l}\text { Calcular a quantidade de quilômetros } \\
\text { que um caminhoneiro autônomo }\end{array}$ & $\begin{array}{l}\text { Bissecção } \\
\text { Secante }\end{array}$ & $\begin{array}{l}\text { Economia } \\
\text { Engenharia }\end{array}$ \\
\hline
\end{tabular}




\begin{tabular}{|l|l|l|}
\hline $\begin{array}{l}\text { precisa se locomover para que tenha o } \\
\text { retorno do seu investimento (compra } \\
\text { de um caminhão). }\end{array}$ & \\
\hline $\begin{array}{l}\text { Análise de algumas variáveis de } \\
\text { processo a fim de proporcionar estudo } \\
\text { e elaboração de padrões de processo } \\
\text { sem interferir nos resultados. }\end{array}$ & Secante & $\begin{array}{l}\text { Planejamento } \\
\text { Controle } \\
\text { Produção. }\end{array}$ \\
\hline $\begin{array}{l}\text { Análise de vazão volumétrica de um } \\
\text { tubo de seção circular. }\end{array}$ & $1 / 3$ de Simpson & $\begin{array}{l}\text { Mecânica dos } \\
\text { Fluidos } \\
\text { fenômenos de } \\
\text { transporte. }\end{array}$ \\
\hline $\begin{array}{l}\text { Cálculo da área atingida pelo } \\
\text { rompimento da barragem. }\end{array}$ & $\begin{array}{l}\text { Integração } \\
\text { Ambiente. }\end{array}$ \\
\hline
\end{tabular}

Fonte: As autoras (2021)

\section{RESULTADOS E DISCUSSÃO}

A partir dos dados coletados foi possível observar os ganhos obtidos. É importante ressaltar que, para que as competências pretendidas sejam adquiridas pelos alunos, é necessário que o professor da disciplina forneça as possibilidades.

Para desenvolver as Competências Técnicas, foi necessário o aluno buscar informações sobre os métodos. Além disso, a professora explanou o conteúdo, porém pôs em prática o princípio da tendência pedagógica centrada na pessoa, onde o aluno é visto como o ponto central do processo de ensino-aprendizagem. Nesse caso, a docente foi facilitadora do processo, e não a detentora de todo o conhecimento. As Competências Técnicas desenvolvidas foram: realizar aproximação de funções numericamente; resolver equações diferenciais numericamente; resolver integrais numericamente; resolver sistemas de equações numericamente; programar em ambiente computacional; e desenvolver o raciocínio lógico para a resolução de problemas.

Já as Competências Transversais adquiridas foram:

- Trabalhar cooperativamente em grupos: visto que os trabalhos foram realizados em equipes e os alunos precisaram lidar com as diferenças. Isso pode ser verificado em seus relatos no diário de bordo, inseridos no Ambiente Virtual de Aprendizagem (Moodle).

- Pensar criticamente: ser capaz de analisar e resolver complexos problemas do mundo real; ser capaz de usar técnicas, habilidades e ferramentas científicas e técnicas modernas necessárias para a prática profissional; e ainda desenvolver a capacidade de identificar e resolver problemas de ciências aplicadas. Estes puderam ser observados quando os alunos foram incentivados a pesquisar sobre os conteúdos relacionados ao tema do problema, e foram capazes de buscarem sozinhos as soluções.

- Demonstrar habilidade de comunicação versátil e efetiva, tanto verbal quanto escrita: isso foi oportunizado aos alunos através da comunicação interna à equipe, a comunicação com professores na busca por conhecimento, na escrita no diário de bordo, na escrita e apresentação dos relatórios em seminários.

- Ter capacidade de síntese: foi trabalhada quando o aluno compreendeu o problema real e foi capaz de criar um modelo representativo em papel. Além disso, foi capaz 
de gerar um relatório e uma apresentação em um tempo determinado, de forma compacta, com dimensão inferior ao tamanho do trabalho realizado.

Pelo fato de atividades avaliativas individuais terem sido realizadas em paralelo ao desenvolvimento das soluções dos problemas, foi possível para o aluno demonstrar os conhecimentos adquiridos quando do esforço individual no processo, visto que a avaliação em equipe já estava sendo observada de outras formas.

Espera-se que a partir do desenvolvimento desta metodologia alternativa de ensinoaprendizagem o aluno utilize o conteúdo, os conhecimentos e as habilidades intelectuais adquiridas/ aprendidas para tornarem-se contínuos aprendizes e que tenham uma visão ampla, necessária para compreender o impacto das soluções em um contexto global e social.

\section{CONCLUSÕES}

O trabalho aqui descrito cumpriu com o objetivo apresentar experiências exitosas verificadas no curso de graduação em Engenharia de Produção do Instituto Federal do Espírito Santo - campus Cariacica, utilizando tecnologias digitais e metodologias ativas na disciplina de Cálculo Numérico.

Foi possível observar que o trabalho aqui demonstrado atendeu a todos os requisitos de um método de ensino Problem-Based Learning, visto que: 1) apresentou o problema antes da teoria; 2) utilizou-se de problemas baseados no mundo real; 3) proporcionou aos alunos elaborarem um produto final concreto (o qual neste caso foi um relatório); 4) as professoras envolvidas implementaram o processo de ensino-aprendizagem centrado no estudante; 5) estimulou a aprendizagem em equipe; 6) lançou mão do papel do professor como facilitador do processo; e 7) utilizou-se do ciclo PBL para a resolução dos problemas, por parte dos alunos.

O contexto de pandemia, de maneira inesperada trouxe restrições especialmente para alunos. Portanto, as limitações deste trabalho foram especialmente os recursos tecnológicos necessários para a ministração da disciplina a distância, visto que para alguns alunos são barreiras que os impossibilitam de assistir aulas, participar de reuniões, dentre outros. As principais dificuldades encontradas pelos alunos, além das tecnológicas, foram o trabalho em equipe; a comunicação dentro da equipe e o controle do tempo. Destaca-se que a leitura dos artigos nos quais os alunos encontraram os problemas os levou para um caminho mais rápido até a solução.

Para a próxima edição da disciplina as professoras irão apresentar problemas reais, em vez de deixarem os alunos procurarem os artigos. Desta forma, espera-se que desenvolvam com maior afinco a busca pela construção do conhecimento e, consequentemente, as melhores soluções para os problemas. Para sanar as dificuldades encontradas pelos alunos em termos de trabalho em equipe, comunicação e controle do tempo, as professoras irão inserir os princípios de Gestão de Projetos, bem como ferramentas e técnicas de planejamento e acompanhamento de projeto, nas aulas iniciais. Estes alunos são do $5^{\circ}$ período e a disciplina de Gestão de Projetos, neste curso, é ministrada no $7^{0}$ período.

Também para uma futura edição da disciplina pretende-se inserir uma avaliação dos seminários por parte de outros professores do curso, e ainda elaborar e aplicar um questionário para que os alunos respondam ao final da disciplina, facilitando a extração e análise dos dados, podendo trazer retornos quantitativos aos mesmos, de modo que possamos comparar alguns indicadores de efetiva aprendizagem. 
Finalmente, espera-se com este trabalho incentivar outros docentes a propor atividades diversificadas relacionando os conteúdos de cálculo numérico com outras áreas de interesse dos alunos, diretamente relacionadas a sua área de formação, de forma a ampliar suas possibilidades de resolução de problemas e análise crítica.

\section{Agradecimentos}

As autoras agradecem o apoio financeiro e institucional do Ifes campus Cariacica e da Reitoria do Ifes.

\section{REFERÊNCIAS}

ABENGE. Inovação na educação em engenharia proposta de diretrizes para o curso de engenharia- abenge, 2018.

Aquere, A. L., Mesquita, D., Lima, R. M., Monteiro, S. B. S., \& Zindel, M. (2012). Coordination of Student Teams focused on Project Management Processes. International Journal of Engineering Education, 28(4), 859-870.

Barrows, H. S. Problem Based Learning Initiative. IL: Southern Illinois University School of Medicine. Disponível em: <http://www.siumed.edu/dme >. Acesso em: 8 de maio de 2021.

Boud, D.; Feletti, G. Changing-problem learning. In BOUD, D.; FELETTI, G. (Eds.). The challenge of problem-based learning. London, Kogan Page, p. 1-14, 1999.

Christie, M., \& de Graaff, E. The philosophical and pedagogical underpinnings of Active Learning in Engineering Education. European Journal of Engineering Education, 42(1), 516. doi:10.1080/03043797.2016.1254160. 2017

Duch, B. J.; Groh, S. E.; Allen, D. E. Why problem-based learning? A case study of institutional change in undergraduate education. In: DUCH, B. J.; GROH, S. E.; ALLEN,D. E. The power of problem-based learning. Virginia: Stylus, p. 3-11, 2001.

Hmelo-Silver, C.E., Problem-based Learning: what and how do students learn? Educacional Psychology Review, v. 6, n. 3, p. 235-266, 2004.

Kolmos, A., Dahms, M. e Du, X., Engineering: Issues, Challenges and Opportunities for Development, United Nations Educational, Scientific and Cultural Organization - UNESCO, Paris, France, 2010.

Lima, R. M., Carvalho, D., Sousa, R. M., Alves, A., Moreira, F., Mesquita, D., \& Fernandes, S. Estrutura de Gestão para Planejamento e Execução de Projetos Interdisciplinares de Aprendizagem em Engenharia. In L. C. d. Campos, E. A. T. Dirani e A. L. Manrique (Eds.), Educação em Engenharia: Novas Abordagens (pp. 87-121). São Paulo, Brasil: EDUC - Editora da PUC-SP. 2011.

Lima, R., Mesquita, Amorim M., Jonker G., e Flores M. A, "An Analysis of Knowledge Areas in Industrial Engineering and Management Curriculum,"International Journal of Industrial Engineering and Management, vol. 3, pp. 75-82, 2012.

Lima, R.M., Dinis-Carvalho, J., Sousa, R. M., Alves, A. C., Moreira, F., Fernandes, S., \& Mesquita, D. (2017). Ten years of projectbased learning (PBL) in industrial engineering and management at 
the University of Minho. In PBL in Engineering Education: International Perspectives on Curriculum Change. https://doi.org/10.1007/978-94-6300-905-8

Maurer, H. Best practices in problem-based learning. In J. Ishiyama, W. J. Miller, e E. Simon (Eds.), Handbook on teaching and learning in political science and international relations (pp. 369383). Cheltenham: Edward Elgar. 2015.

Quadros, R. e Villas-Boas, V. Aprendizagem Ativa no Ensino Médio: Uma Proposta para o Ensino de Grandezas Físicas e Unidades de Medida Utilizando Casos de Ensino. Scientia Cum Industria, v. 8, no. 3, pp. 17-21, 2020.

WHITE, H. B. Dan tries problem-based learning: A case study. In: RICHLIN, L. (Eds.). To Improve the Academy. Stillwater, OK: New Forums Press and the Professional and Organizational Network in Higher Education, p. 75-91, 1996.

\begin{abstract}
The present paper aims to present and discuss the importance of the numerical analysis discipline for undergraduate engineering students, especially in subjects related to their studies areas. This study aiming to show the potential of this tools/ methods to obtain an approximate solution of mathematical problems. These methods are applying to problems that do not have an exact solution and must be solved numerically. In particular, this article presents successful experiences in the Production Engineering undergraduate course in Federal Institute of Espírito Santo - campus Cariacica. Most of the problems in basic and specific disciplines of engineering undergraduate courses arise from the observation of society, industry, nature, among other aspects, and such problems can be described through mathematical models modeled numerically. In this paper, some themes related to numerical analysis and other basic disciplines of engineering are presented. Examples: fluid mechanics and transport phenomena; analysis of environmental data, related to the discipline of environmental sciences; and economic and financial evaluation of engineering projects. In all projects developed, alternative activities were carried out with the students using digital technologies and active methodologies, to ensure that students remained motivated and stimulated to develop the proposed activities. In this way, this work aimed to encourage other teachers to propose diversified activities connecting numerical analysis with other areas of interest to students, in order to expand their problem-solving possibilities and their critical analysis.
\end{abstract}

Keywords: Numerical analysis. Engineering Education. Active methodologies. ProblemBased Learning. 